\title{
Stationarity and Invertibility of a Dynamic Correlation Matrix ${ }^{\star \star}$
}

\author{
Christian M. Hafner ${ }^{1}$ and Michael McAleer ${ }^{2^{*}}$ \\ ${ }^{1}$ ISBA and CORE \\ Université Catholique de Louvain, Belgium \\ ${ }^{2}$ Department of Quantitative Finance \\ National Tsing Hua University, Taiwan \\ *michael.mcaleer@gmail.com
}

\begin{abstract}
One of the most widely-used multivariate conditional volatility models is the dynamic conditional correlation (or DCC) specification. However, the underlying stochastic process to derive DCC has not yet been established, which has made problematic the derivation of regularity conditions, such as stationarity and invertibility, and asymptotic properties of the Quasi-Maximum Likelihood Estimators (QMLE). To date, the statistical properties of the QMLE of the DCC parameters have been derived under highly restrictive and unverifiable regularity conditions, which essentially leads to proof by assumption. The paper shows that the DCC model can be obtained from a vector random coefficient moving average process, and derives the stationarity and invertibility conditions of the DCC model. The derivation of DCC from a vector random coefficient moving average process raises three important issues: (i) demonstrates that DCC is, in fact, a dynamic conditional covariance model of the returns shocks rather than a dynamic conditional correlation model; (ii) provides the motivation, which is presently missing, for standardization of the conditional covariance model to obtain the conditional correlation model; and (iii) shows that the appropriate univariate conditional volatility model for DCC is based on the standardized shocks rather than the returns shocks. The derivation of the regularity conditions, especially stationarity and invertibility, should subsequently lead to a solid statistical foundation for the estimates of the DCC parameters.
\end{abstract}

Keywords: Eigen values and eigenvectors, dynamic conditional correlation, dynamic conditional covariance, vector random coefficient moving average, stationarity, invertibility, asymptotic properties.

\section{Introduction}

In a prescient and innovative paper, Laloux et al. (1999) examined dynamic correlation matrices for portfolios of financial assets for purposes of risk management based on eigen values and eigenvectors. The primary purpose of their contribution was to test whether the correlation matrix was purely random. A serious question remains as to how to estimate the time-varying or dynamic correlations when the null hypothesis of randomness is rejected. The primary purpose of this paper is to analyse a subsequent development on dynamic correlations based on the financial econometrics literature on multivariate conditional correlations and conditional volatility.

There has been little research on analyzing dynamic correlations, possibly because of the difficulty in establishing regularity conditions for the internal consistency of models, and the subsequent proofs of asymptotic properties of the estimators, namely consistency and asymptotic normality, for purposes of valid statistical inference. Among multivariate conditional volatility models, the dynamic

\footnotetext{
** The authors are most grateful to Massimiliano Caporin, Guillaume Gaetan Martinet and Yuk Tse for helpful comments and suggestions. For financial support, the second author wishes to acknowledge the Australian Research Council and the National Science Council, Ministry of Science and Technology (MOST), Taiwan. McAleer is University Distinguished Research Professor at the Institute for Social and Economic Sciences (ISES), Dhurakij Pundit University (DPU), Bangkok, Thailand.
} 
conditional correlation (or DCC) specification of Engle (2002) is one of the most widely used in practice. The basic DCC modelling approach has been as follows: (i) estimate the univariate conditional variances using the GARCH(1,1) model of Bollerslev (1986), which are based on the returns shocks; and (ii) estimate the conditional correlation matrix of the standardized residuals. The first step is arbitrary as the conditional variances could just as easily be based on the standardized residuals (that is, the returns shocks standardized by the conditional variance), as will be shown in Section 4 below.

A similar comment applies to the varying conditional correlation model of Tse and Tsui (2002), where the first stage is based on a standard conditional volatility model using returns shocks. The second stage is slightly different from the DCC formulation as the conditional correlations are defined appropriately. However, no regularity conditions are presented, and hence no statistical properties are given.

The DCC model has been analyzed critically in a number of papers as its underlying stochastic process has not yet been established, which has made problematic the derivation of the asymptotic properties of the Quasi-Maximum Likelihood Estimators (QMLE). To date, the statistical properties of the QMLE of the DCC parameters have been derived under highly restrictive and unverifiable regularity conditions, which in essence amounts to proof by assumption.

This paper shows that the DCC specification can be obtained from a vector random coefficient moving average process, and derives the conditions for stationarity and invertibility of the DCC model. The derivation of regularity conditions should subsequently lead to a solid statistical foundation for the estimates of the DCC parameters.

The derivation of DCC from a vector random coefficient moving average process raises three important issues: (i) demonstrates that DCC is, in fact, a dynamic conditional covariance model of the returns shocks rather than a dynamic conditional correlation model; (ii) provides the motivation, which is presently missing, for standardization of the conditional covariance model to obtain the conditional correlation model; and (iii) shows that the appropriate conditional volatility model for DCC is based on the standardized shocks rather than the returns shocks.

The remainder of the paper organized is as follows. In Section 2, the standard conditional volatility model is derived from a random coefficient autoregressive process to provide a background for the remainder of the paper. In Section 3, the DCC model is discussed. Section 4 presents a vector random coefficient moving average process, from which DCC is derived in Section 5. The conditions for stationarity and invertibility of DCC are given in Section 6. Some concluding comments are given in Section 7.

\section{Random Coefficient Autoregressive Process}

Consider the following a random coefficient autoregressive process of order one:

$$
\varepsilon_{t}=\phi_{t} \varepsilon_{t-1}+\eta_{t}
$$

where

$$
\begin{aligned}
& \phi_{t} \sim i i d(0, \alpha), \\
& \eta_{t} \sim i i d(0, \omega), \text { independent of }\left\{\phi_{t}\right\} .
\end{aligned}
$$

The ARCH(1) model of Engle (1982) can be derived as (see Tsay (1987)): 
$h_{t}=E\left(\varepsilon_{t}^{2} \mid I_{t-1}\right)=\omega+\alpha \varepsilon_{t-1}^{2}$

where $h_{t}$ is conditional volatility, and $I_{t-1}$ is the information set at time $t-1$. The use of an infinite lag length for the random coefficient autoregressive process leads to the GARCH model of Bollerslev (1986).

The scalar and diagonal BEKK models of Baba et al. (1985) and Engle and Kroner (1995), which lead to dynamic multivariate covariance models, can be derived from a vector random coefficient autoregressive process (see McAleer et al. (2008)). As the statistical properties of vector random coefficient autoregressive processes are well known, the statistical properties of the parameter estimates of the ARCH, GARCH, scalar BEKK and diagonal BEKK models are straightforward to establish.

\section{DCC Specification}

Let the conditional mean of financial returns be given as:

$y_{t}=E\left(y_{t} \mid I_{t-1}\right)+\varepsilon_{t}$

where $y_{t}=\left(y_{1 t, \ldots,} y_{m t}\right)^{\prime}, y_{i t}=\Delta \log P_{i t}$ represents the log-difference in stock prices $\left(P_{i t}\right), i=$ $1, \ldots, m, I_{t-1}$ is the information set at time $t-1$, and $\varepsilon_{t}$ is conditionally heteroskedastic. Without distinguishing between dynamic conditional covariances and dynamic conditional correlations, Engle (2002) presented the DCC specification as:

$Q_{t}=(1-\alpha-\beta) \bar{Q}+\alpha \eta_{t-1} \eta_{t-1}^{\prime}+\beta Q_{t-1}$

where $\bar{Q}$ is assumed to be positive definite with unit elements along the main diagonal, the scalar parameters are assumed to satisfy the stability condition, $\alpha+\beta<1$, the standardized shocks, $\eta_{t}=\left(\eta_{1 t, \ldots,} \eta_{m t}\right)^{\prime}$, which are not necessarily iid, are given as $\eta_{i t}=\varepsilon_{i t} / \sqrt{h_{i t}}$, with $\varepsilon_{t}=D_{t} \eta_{t}$, and $D_{t}$ is a diagonal matrix with typical element $\sqrt{h_{i t}}, i=1, \ldots, m$.

If $m$ is the number of financial assets, the multivariate definition of the relationship between $\varepsilon_{t}$ and $\eta_{t}$ is now given as $\varepsilon_{t}=D_{t} \eta_{t}$.

Define the conditional covariance matrix of ${ }^{\varepsilon_{t}}$ as $Q_{t}$. As the $m \times 1$ vector, ${ }^{\eta_{t}}$, is assumed to be iid for all $m$ elements, the conditional correlation matrix of $\eta_{t}$, which is equivalent to the conditional correlation matrix of $\eta_{t}$, is given by $\Gamma_{t}$. Therefore, the conditional expectation of $\varepsilon_{t}$ is defined as:

$$
Q_{t}=D_{t} \Gamma_{t} D_{t} .
$$


Equivalently, the conditional correlation matrix, ${ }^{\Gamma_{t}}$, can be defined as:

$$
\Gamma_{t}=D_{t}^{-1} Q_{t} D_{t}^{-1}
$$

Equation (5) is useful if a model of $\Gamma_{t}$ is available for purposes of estimating ${ }^{t}$, whereas equation (6) is useful if a model of $Q_{t}$ is available for purposes of estimating $\Gamma_{t}$.

In view of equations (5) and (6), as the matrix in equation (4) does not satisfy the definition of a correlation matrix, Engle (2002) uses the following standardization:

$$
R_{t}=\left(\operatorname{diag}\left(Q_{t}\right)\right)^{-1 / 2} Q_{t}\left(\operatorname{diag}\left(Q_{t}\right)\right)^{-1 / 2}
$$

There is no clear explanation given in Engle (2002) for the standardization in equation (7) or, more recently, in Aielli (2013), especially as it does not satisfy the definition of a correlation matrix, as given in equation (6). The standardization in equation (7) might make sense if the matrix $Q_{t}$ were the conditional covariance matrix of ${ }^{\varepsilon_{t}}$ or $\eta_{t}$, though this is not made clear. It is worth noting that the unconditional covariance matrix of ${ }^{\varepsilon_{t}}$ is not analytically tractable as its stochastic process is not stated explicitly.

Despite the title of the paper, Aielli (2013) also does not provide any stationarity conditions for the DCC model, and does not mention invertibility. Indeed, in the literature on DCC, it is not clear whether equation (4) refers to a conditional covariance or a conditional correlation matrix. In this respect, some caveats regarding DCC are given in Caporin and McAleer (2013).

\section{Vector Random Coefficient Moving Average Process}

Marek (2005) proposed a linear moving average model with random coefficients (RCMA), and established the conditions for stationarity and invertibility. In this section, we extend existing theoretical results by deriving the stationarity and invertibility conditions of a vector random coefficient moving average process.

Consider a univariate random coefficient moving average process given by:

$$
\varepsilon_{t}=\theta_{t} \eta_{t-1}+\eta_{t}
$$

where $\eta_{t} \sim$ iid $(0, \omega)$. The sequence $\left\{\theta_{t}\right\}$ is supposed to be independent of $\eta_{t-1}, \eta_{t}, \eta_{t+1}, \ldots$, which is called the future independence condition, with a mean zero and variance $\alpha$. It is also assumed to be measurable w.r.t. ${ }^{I_{t}}$, where $I_{t}$ is the information set generated by the r.v. $\left\{\varepsilon_{t}, \varepsilon_{t-1, \ldots}\right\}$. Furthermore, assume that the process $\left\{\varepsilon_{t}\right\}$ is stationary and invertible such that $\eta_{t} \in I_{t}$.

Without the measurability assumption on $\left\{\theta_{t}\right\}$ it would be difficult to obtain results on the invertibility of the model, which is essential for purposes of deriving the likelihood equation for estimating the associated parameters. However, an important special case of the model arises when $\left\{\theta_{t}\right\}_{\text {is }}$ iid, that is, not measurable with respect to ${ }^{I_{t}}$, in which case the conditional and unconditional expectations of ${ }^{\varepsilon_{t}}$ are zero, and the conditional variance of ${ }^{\varepsilon_{t}}$ is given by: 
$h_{t}=E\left(\varepsilon_{t}^{2} \mid I_{t-1}\right)=\omega+\alpha \eta_{t-1}^{2}$

which differs from the $\mathrm{ARCH}(1)$ model in equation (2) in that the returns shock is replaced by the standardized shock. As $\eta_{t} \sim$ iid $(0, \omega)$, the unconditional variance of $\varepsilon_{t}$ is given as:

$$
E\left(h_{t}\right)=(1+\alpha) \omega .
$$

The use of an infinite lag length for the random coefficient moving average process in equation (8), with appropriate restrictions on ${ }^{\theta_{t}}$, would lead to a generalized ARCH model that differs from the GARCH model of Bollerslev (1986) as it would replace the returns shock with a standardized shock.

The univariate $\mathrm{ARCH}(1)$ model in equation (9) is contained in the family of GARCH models proposed by Hentschel (1995), and the augmented GARCH model class of Duan (1997).

It can be shown from the results in Marek (2005) that a sufficient condition for stationarity is that the vector sequence $v_{t}=\left(\eta_{t}, \theta_{t} \eta_{t-1}\right)^{\prime}$ is stationary. Moreover, by Lemma 2.1 of Marek (2005), a sufficient condition for invertibility is that:

$$
E\left[\log \left|\theta_{t}\right|\right]<0
$$

The stationarity of $v_{t}=\left(\eta_{t}, \theta_{t} \eta_{t-1}\right)^{\prime}$ and the invertibility condition in equation (10) are new results for the novel univariate $\mathrm{ARCH}(1)$-type model given in equation (9), as well as its direct extension to associated GARCH-type models.

Extending the analysis given above to the multivariate case and to a vector random coefficient moving average (RCMA) model of order $p$, we can derive a special case of $\operatorname{DCC}(p, q)$, namely $\operatorname{DCC}(p, 0)$, as follows:

$$
\varepsilon_{t}=\sum_{j=1}^{p} \theta_{j t} \eta_{t-j}+\eta_{t}
$$

where $\varepsilon_{t}$ and $\eta_{t}$ are both $m \times 1$ vectors and $\theta_{j t}, j=1, \ldots, p$ are random $m \times m$ matrices, independent of $\eta_{t-1}, \eta_{t}, \eta_{t+1}, \ldots$. Under the following Assumption 1, it is possible to derive the conditional covariance matrix of ${ }^{\varepsilon_{t}}$ in equation(11):

Assumption 1:

$$
E\left(\eta_{t} \mid I_{t-1}\right)=0, E\left(\eta_{t} \eta_{t}{ }^{\prime} \mid I_{t-1}\right)=\Omega
$$

(ii) The random coefficient matrices ${ }^{\theta_{j t}}$ have the following properties, for all $j=1, \ldots, p, t=1, \ldots, T$ :

$$
E\left(\theta_{j t} \mid I_{t-1}\right)=0, \quad E\left(\theta_{j t, k l} \theta_{j t, m n}{ }^{\prime} \mid I_{t-1}\right)=A_{j, k l} A_{j, m n}{ }^{\prime}, \text { and } E\left(\theta_{j t, k l} \theta_{i s, m n}{ }^{\prime} \mid I_{t-1}\right)=0, i \neq j, \text { and/or } s \neq t
$$


Using the conditions in Proposition 1 of McAleer et al. (2008), it follows that the conditional covariance matrix is given by:

$$
\begin{aligned}
& H_{t}=E\left(\varepsilon_{t} \varepsilon_{t} \mid I_{t-1}\right)=\Omega+\sum_{j=1}^{p} A_{j} \eta_{t-j} \eta_{t-j}^{\prime} A_{j}^{\prime} \\
& E\left(\operatorname{vec}\left(H_{t}\right)\right)=\left(I_{m}+\sum_{j=1}^{p} A_{j} \otimes A_{j}\right) \operatorname{vec}(\Omega)
\end{aligned}
$$

This approach can easily be extended to include autoregressive terms. For example, in a model analogous to $\operatorname{GARCH}(p, q)$, namely:

$$
H_{t}=\Omega+\sum_{i=1}^{p} A_{i} \eta_{t-i} \eta_{t-i}^{\prime} A_{i}{ }^{\prime}+\sum_{j=1}^{q} B_{j} H_{t-j} B_{j}{ }^{\prime}
$$

where the parameter matrices $B_{j}$ are such that the maximum eigenvalue of $\sum_{j=1}^{q} B_{j} \otimes B_{j}$ is smaller than one in modulus, it follows that:

$$
E\left(\operatorname{vec}\left(H_{t}\right)\right)=\left(I_{m}-\sum_{j=1}^{q} B_{j} \otimes B_{j}\right)^{-1}\left(I_{m}+\sum_{j=1}^{p} A_{j} \otimes A_{j}\right) \operatorname{vec}(\Omega)
$$

The derivation given above shows that, as compared with the standard DCC formulation, which is not based on any known stochastic process, the formulation given above permits straightforward computation of the unconditional variances and covariances.

It should also be noted that in Aielli's (2013) variation of the standard DCC model, it is possible to calculate the unconditional expectation of the $Q_{t}$ matrix, as in equation (4), but this is not equal to the unconditional covariance matrix of $\varepsilon_{t}$, which is analytically intractable. This is an additional advantage of using the vector random coefficient moving average process given in equation (11).

\section{One Line Derivation of DCC}

If $\theta_{j t}$ in equation (11) is given as:

$\theta_{j t}=\lambda_{j t} I_{m}$, with $\lambda_{j t} \sim \operatorname{iid}\left(0, \alpha_{j}\right), \quad j=1, \ldots, p$,

where $\lambda_{j t}$ is a scalar random variable, then the conditional covariance matrix can be shown to be:

$$
H_{t}=E\left(\varepsilon_{t} \varepsilon_{t}^{\prime} \mid I_{t-1}\right)=\Omega+\sum_{j=1}^{p} \alpha_{j} \eta_{t-j} \eta_{t-j}^{\prime}
$$


The DCC model in equation (4) is obtained by letting $p \rightarrow \infty$, setting $\alpha_{j}=\alpha \beta^{j-1}$, and standardizing $H_{t}$ to obtain a conditional correlation matrix. For the case $p=1$ in equation (12), the appropriate univariate conditional volatility model is given in equation (9), which uses the standardized shocks, rather than in equation (2), which uses the returns shocks.

The derivation of DCC in equation (12) from a vector random coefficient moving average process is important as it: (i) demonstrates that DCC is, in fact, a dynamic conditional covariance model of the returns shocks rather than a dynamic conditional correlation model; (ii) provides the motivation, which is presently missing, for standardization of the conditional covariance model to obtain the conditional correlation model; and (iii) shows that the appropriate ARCH or GARCH model for DCC is be based on the standardized shocks rather than the returns shocks.

\section{Derivation of Stationarity and Invertibility}

The formulation of DCC given in the previous section is more natural than the standard treatment as it can be derived from an underlying stochastic process, and can be also analyzed in terms of properties such as stationarity and moments.

This section derives the stationarity and invertibility conditions for the DCC model in Theorem 1, based on Assumption 2:

Assumption 2. $E\left[\log \left\|\Theta_{t-k}\right\|\right]<\sqrt{p m}$

where $\left\|\Theta_{t}\right\|$ is the Frobenius norm, and $\Theta_{t}$ is given by:

$\Theta_{t}=\left(\begin{array}{cccc}-\theta_{1 t} & -\theta_{2 t} & \ldots & -\theta_{p t} \\ 1 & 0 & \ldots & 0 \\ \cdot & . & . & \cdot \\ 0 & \ldots & 1 & 0\end{array}\right)$

Theorem 1. A sufficient condition for stationarity is that the vector sequence:

$v_{t}=\left(\eta_{t}, \theta_{1 t} \eta_{t-1}, \ldots, \theta_{p t} \eta_{t-p}\right)$

is stationary. Furthermore, under Assumption 2, the vector random coefficient moving average process, ${ }^{\varepsilon_{t}}$, is invertible.

Proof: The proof of stationarity is similar to that given above for the univariate random coefficient moving average process. For invertibility, note that: 
$\eta_{t}=\varepsilon_{t}-\sum_{j=1}^{p} \theta_{j t} \eta_{t-j}$

which can be written as:

$\tilde{\eta}_{t}=\Theta_{t} \tilde{\eta}_{t-1}+\tilde{\varepsilon}_{t}$

where

$\tilde{\eta}_{t}=\left(\eta_{t}, \eta_{t-1}, \ldots, \eta_{t-p+1}\right)^{\prime}$ and $\tilde{\varepsilon}_{t}=\left(\varepsilon_{t}, \varepsilon_{t-1}, \ldots, \varepsilon_{t-p+1}\right)^{\prime}$

Hence,

$\tilde{\eta}_{t}=\sum_{j=0}^{n-1}\left(\prod_{k=1}^{j} \Theta_{t-k+1}\right) \widetilde{\varepsilon}_{t-j}+\left(\prod_{k=0}^{n-1} \Theta_{t-k}\right) \tilde{\eta}_{t-n}$

Now let:

$\tilde{\eta}_{t}^{(n)}=\sum_{j=0}^{n}\left(\prod_{k=1}^{j} \Theta_{t-k+1}\right) \widetilde{\varepsilon}_{t-j}$

Consider

$$
\begin{aligned}
& \frac{1}{n} \log \frac{1}{\sqrt{p m}}\left\|\tilde{\eta}_{t}-\tilde{\eta}_{t}^{n}\right\|=\frac{1}{n} \log \frac{1}{\sqrt{p m}}\left\|\left(\prod_{k=1}^{n-1} \Theta_{t-k}\right) \tilde{\eta}_{t-n}\right\| \\
& \leq \frac{1}{n} \log \frac{1}{\sqrt{p m}}\left\|\prod_{k=1}^{n-1} \Theta_{t-k}\right\|+\frac{1}{n} \log \frac{1}{\sqrt{p m}}\left\|\tilde{\eta}_{t-n}\right\| \\
& \leq \frac{1}{n} \sum_{k=1}^{n} \log \frac{1}{\sqrt{p m}}\left\|\Theta_{t-k}\right\|+\frac{1}{n} \log \frac{1}{\sqrt{p m}}\left\|\tilde{\eta}_{t-n}\right\|
\end{aligned}
$$

$\underset{\text { a.s. }}{\longrightarrow} E \log \frac{1}{\sqrt{p m}}\left\|\Theta_{t-k}\right\|<0$

as $E \log \left\|\Theta_{t-k}\right\|<\sqrt{p m}$, by assumption. This implies that $\eta_{t}-\eta_{t}^{n} \underset{\text { a.s. }}{\longrightarrow} 0$ and, hence, $\eta_{t}$ is asymptotically measurable with respect to $\left\{\varepsilon_{t-1}, \varepsilon_{t-2}, \cdots\right\}$, and $\varepsilon_{t}$ is invertible. 
Note that a sufficient condition for equation (13) is that:

$$
\sum_{j=1}^{p} E\left\|\theta_{j t}\right\|^{2}<m
$$

$$
\begin{aligned}
& \text { as } \quad E \log \frac{1}{\sqrt{p m}}\left\|\Theta_{t-k}\right\| \leq \log E \frac{1}{\sqrt{p m}}\left\|\Theta_{t-k}\right\| \\
& =\log E \frac{1}{\sqrt{p m}} \sqrt{\sum_{j=1}^{p}\left\|\theta_{j t}\right\|^{2}+(p-1) m} \\
& =\log E \sqrt{\frac{1}{p m} \sum_{j=1}^{p}\left\|\theta_{j t}\right\|^{2}+(p-1) / p} \\
& \leq \log \sqrt{\frac{1}{p m} \sum_{j=1}^{p} E\left\|\theta_{j t}\right\|^{2}+(p-1) / p} \\
& <0 .
\end{aligned}
$$

The condition given in equation (14) may be easier to check in practice than the condition given in equation (13).

For the special case $\theta_{j t}=\lambda_{j t} I_{m}$, with $\lambda_{j t} \sim \operatorname{iid}\left(0, \alpha_{j}\right), j=1, \ldots, p$, discussed in Section 5 above, the condition in equation (14) simplifies to the well-known condition on the long-run persistence to returns shocks, namely:

$$
\sum_{j=1}^{p} E \lambda_{j t}^{2}=\sum_{j=1}^{p} \alpha_{j}<1
$$

\section{Conclusion}

The paper was concerned with one of the most widely-used multivariate conditional volatility models, namely the dynamic conditional correlation (or DCC) specification. As the underlying stochastic process to derive DCC has not yet been established, this has made problematic the derivation of the asymptotic properties of the Quasi-Maximum Likelihood Estimators (QMLE). To date, the statistical properties of the QMLE of the DCC parameters have been derived under highly restrictive and unverifiable regularity conditions.

The paper showed that the DCC specification could be obtained from a vector random coefficient moving average process, and derived the stationarity and invertibility conditions of the DCC model. The derivation of the regularity conditions should eventually lead to a solid foundation for the statistical analysis of the estimates of the DCC parameters.

The derivation of DCC from the vector random coefficient moving average process demonstrated that DCC is, in fact, a dynamic conditional covariance model of the returns shocks rather than a dynamic 
conditional correlation model. Moreover, the derivation provided the motivation, which is presently missing, for standardization of the conditional covariance model to obtain the conditional correlation model. The standardization of the estimated DCC models in practice does not satisfy the definition of a correlation matrix, which has always been problematic in interpreting the DCC model.

The derivation also showed that the appropriate univariate conditional volatility model for DCC is based on the standardized shocks rather than the returns shocks. The derivation of regularity conditions should subsequently lead to a solid statistical foundation for the QMLE of the appropriate univariate specifications that underlie the DCC model.

\section{References}

Aielli, G.P. (2013), Dynamic conditional correlations: On properties and estimation, Journal of Business and Economic Statistics, 31, 282-299.

Baba, Y., R.F. Engle, D. Kraft and K.F. Kroner (1985), Multivariate simultaneous generalized ARCH. Unpublished manuscript, Department of Economics, University of California, San Diego, CA, USA.

Bollerslev, T. (1986), Generalised autoregressive conditional heteroscedasticity, Journal of Econometrics, 31, 307-327.

Caporin, M. and M. McAleer (2013), Ten things you should know about the dynamic conditional correlation representation, Econometrics, 1(1), 115-126.

Duan, J.-C. (1997), Augmented GARCH(p,q) process and its diffusion limit, Journal of Econometrics, 79, 97-127.

Engle, R.F. (1982), Autoregressive conditional heteroscedasticity with estimates of the variance of United Kingdom inflation, Econometrica, 50, 987-1007.

Engle, R. (2002), Dynamic conditional correlation: A simple class of multivariate generalized autoregressive conditional hereoskedasticity models, Journal of Business and Economic Statistics, 20, 339-350.

Engle, R.F. and K.F. Kroner (1995), Multivariate simultaneous generalized ARCH, Econometric Theory, 11, 122-150.

Hentschel, L. (1995), All in the family: Nesting symmetric and asymmetric GARCH models, Journal of Financial Economics, 39, 71-104.

Laloux, L., P. Cizeau, J.-P. Bouchaud amd M. Potters (1999), Noise dressing of financial correlation matrices, Physical Review Letters, 83(7), 1467-1470.

Marek, T. (2005), On invertibility of a random coefficient moving average model, Kybernetika, 41(6), 743-756.

McAleer, M., F. Chan, S. Hoti and O. Lieberman (2008), Generalized autoregressive conditional correlation, Econometric Theory, 24(6), 1554-1583.

Tsay, R.S. (1987), Conditional heteroscedastic time series models. Journal of the American Statistical Association, 82, 590-604. 
Tse, Y.K. and A.K.C. Tsui (2002), A multivariate GARCH model with time-varying correlations, Journal of Business and Economic Statistics, 20, 351-362. 